\title{
Simulating and Analysing the Impact of Routing Protocols on Different Parameters of WSNs
}

\author{
Anish Soni \\ HCTM Technical Campus, Kaithal, Haryana, India \\ Rajneesh Randhawa \\ Punjabi University, Patiala, Punjab, India
}

\begin{abstract}
Energy efficiency of Wireless Sensor Networks has become an essential requirement and is the main issue for researchers. Various routing, data dissemination and energy efficient protocols have been designed for Wireless Sensor Networks where energy issue has been given more stress. Sensors in wireless sensor networks work on battery and have limited energy. Hence, network has limited lifetime. Routing protocol plays a major role in deciding for how much time a network will survive. All routing algorithms tend to increase the lifetime of WSN while maintaining factors like successful and real-time delivery of a message. This paper aims towards studying different categories of routing protocols and finally four hierarchical routing protocols LEACH, EHRP, SEP and FAIR have been simulated. The performance of each routing protocol has been measured on some performance metrics like network lifetime, packets transferred to BS, number of dead nodes etc and finally concluded that how a routing protocol can impact the network lifetime.
\end{abstract}

Keywords: Hierarchical Routing Protocols, Wireless Sensor Networks, LEACH, EHRP, SEP, FAIR, Energy-efficiency, Network Lifetime.

\section{INTRODUCTION}

wireless sensor network consists of large quantity of tiny sensor nodes which are broadly distributed over a geographical area 11]. The main functionality of a sensor node is observing, notifying and recording a particular condition in different locations and to pass the data with cooperation of other nodes to the main location (Sink Node), where the end user can access the data. Sound, humidity, temperature, air-pressure, vibrations etc are such conditions which a sensor network can sense and process. For all such functions to perform efficiently, these nodes depends on a battery because battery replacement is very difficult and expensive in sensor networks after deploying. Since power-efficient circuits and advance networking technologies are available now, so these sensor nodes can stay alive for up to three years even with a 2 AA Battery.

Journal of Technology Management for Growing Economies Vol. 6, No. 1 April 2015 pp. 27-37

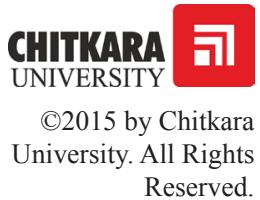


Soni, A.

Randhawa, R.

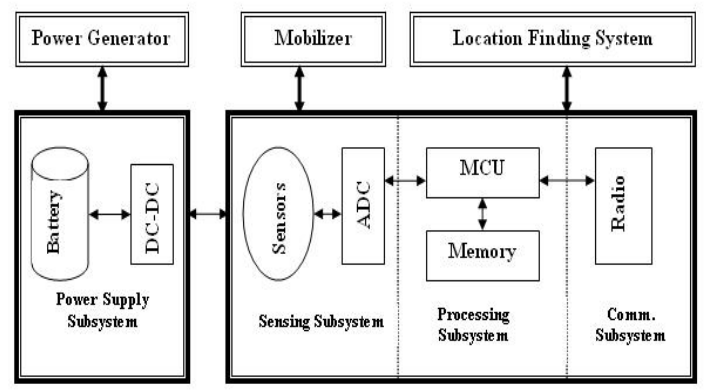

Figure 1: Sensor Node Components

Every Sensor Node is made up of three components for capturing, processing and data transmission. System used for capturing data from the environment is Sensing-Subsystem, System for local data processing is ProcessingSubsystem and System used for data transmission to a central collection point is Communication Subsystem. Except these, sensor node has also mobilizer, location finding system and power generator as shown in Figure 1.When a large number of such sensor nodes deploy to make up a network than it consists of following three elements:

- Sensor Field: Area for the sensor nodes to deploy.

- Sensor Nodes: which actually gather data from surroundings and pass the same to the sink.

- Sink: A special node which receives data from the other nodes, process it and gives results to the end user as shown in figure 2.

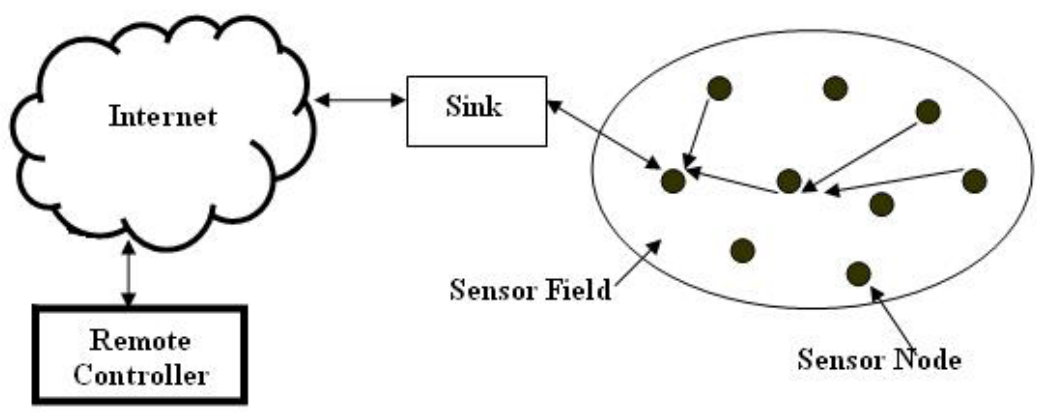

User

Figure 2: Sensor Network Architecture

After deploying in a field, every sensor network faces some common challenges which can lead to the failure of network. These challenges are:

Journal of Technology Management for Growing Economies, Volume 6, Number 1, April 2015 
- Sensor Nodes are resource-poor and have very limited memory, bandwidth, transmission range and CPU power.

- They are capable of storing very limited amount of energy.

- Environmental conditions affects the performance of sensor nodes.

- In a network, where sensor nodes are moving around, network topology changes very frequently.

- Heterogeneity

With all such challenges, it is very difficult for a sensor network to survive for a long period. Therefore, it becomes necessary to make some arrangements in the network to improve its lifetime and other parameters such as total packets transmitted to base station and average energy consumed in given time interval. Various techniques have been developed, so far, for the improvement of such parameters. It has been analysed and simulated in this paper that these parameters can be improved, if a better routing technique, at network layer, is adopted because experiments shown that transmission of data from one node to another consumes much more energy than to process the data at a node [2]. Energy cost of transmitting a single bit of information over a $50 \mathrm{~m}$ is approximately the same as that needed for processing 1500 operations in a sensor node. We simulate hierarchical cluster based routing techniques in MATLAB for a given scenario and found the effect on different parameters.

\section{ROUTING IN WIRELESS SENSOR NETWORKS- DESIGN ISSUES}

Routing in Wireless Sensor Networks becomes very challenging because of some characteristics which are specific to these networks and different from other networks. Resource-poorness of WSN in energy, processing power, and storage capacity make it necessary that all such resources should be managed carefully. Also, in all applications of sensor networks, data flows from multiple source nodes to a particular Base Station. It is also not possible to make a universal addressing scheme for such large numbers of sensor nodes to deploy. So, traditional protocols (IP-based) are not useful in WSN. Also, design of network changes with different applications. Location management is another factor in WSN which is important and cannot be ignored because for accurate data transmission, position of a sensor node must be known and Global Positioning System (GPS) is not feasible to use for the purpose. Finally, data redundancy chances are also high in such networks because the neighbouring nodes can transmit the same data collected from the environment. Considering all such factors, we summarize different design issues [3] in routing into following categories:

Fault-Tolerance: Some of the nodes in sensor network may become non-

Journal of Technology Management for Growing Economies, Volume 6, Number 1, April 2015 
Soni, A.

Randhawa, R.

functional due to lack of energy or some other environmental conditions. In such cases, routing protocols must be capable of finding and linking new routes to the sink node where data is being collected.

Node-Deployment: Nodes in the WSNs can be deployed either manually or scattered randomly depending upon the location and application for which network is designed. In manual placement, nodes are placed manually in the area and data from source node to destination is routed through predetermined path whereas in random placement nodes cannot follow the predetermined path so they follow self-organized(ad-hoc) infrastructure. In that case, distribution of sensor nodes is not uniform, thus, raising the issue of proper selection of cluster head in the network for minimum energy consumption.

Data Delivery Method: Main functionality of sensor nodes in a network is to sense and collect the data from the surroundings and pass it to the base station. This passing of data can be continuous or non-continuous. In continuous model, the data is sent periodically and in non-continuous model it can be event-based or query-based i.e. data will be passed only when an particular event will occur or some query is fired by the sink. Some applications requires combination of both(hybrid) also. Therefore Routing protocols must be designed according to the requirement of data delivery method. It has been observed that for habitat monitoring applications, it is better to use hierarchical protocols because redundant data can be aggregated and thus energy consumption can be reduced in such protocols.

Heterogeneity: All the sensor nodes in a network were assumed to have equal capabilities(energy, computational power etc) in some studies but this is not always desirable because in some applications few nodes have to play some special role while transferring data to the sink nodes. So, this heterogeneous nature of nodes raises some technical issues as per the routing concerns. For example in hierarichal routing protocols, one node is selected as cluster head and this cluster head transfers the aggregated data received from its cluster nodes. Such cluster heads must be having more energy and computational power as compare to normal nodes in the network.

Scalability: To sense and respond to different events in WSNs, routing protocols must be scalable enough because any time, it is possible to add some more nodes in the existing network for an application to run successfully.

Coverage: When we deploy a sensor node in the environment, it obtains only limited view because it can obtain only a limited physical area. Therefore area coverage is also an important design issue in WSNs.

Journal of Technology Management for Growing Economies, Volume 6, Number 1, April 2015 
Energy-Consumption: Each sensor node has to sense, compute and transmit the data to other nodes or sink node. All these operations require a sufficient amount of energy. If any node is having energy level lower than the minimum requirement, it can lead to the topological change in the network or even the failure of network. Therefore, routing protocols must be designed by taking energy consumption factor into consideration and some energy-saving forms of computing and transmitting the data are essential.

\section{ROUTING PROTOCOLS IN WSN}

Since the main objective of this paper is to find the impact of network structure based hierarchical routing protocols on various parameters of sensor networks, we described here a brief introduction about different categories of routing protocols used in WSNs. Routing protocols can be categorize in four ways [4] as shown in Figure 3.

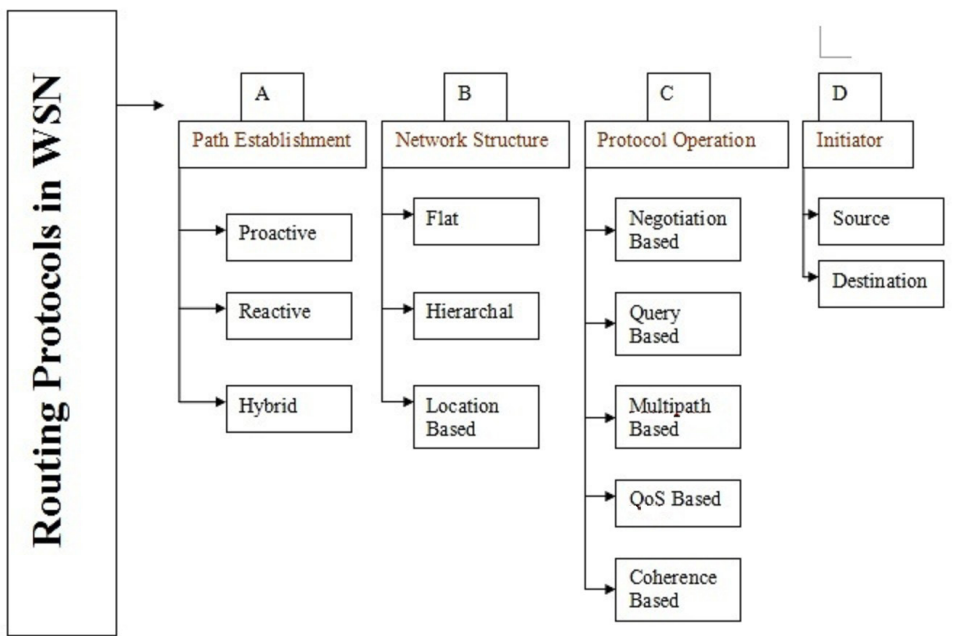

Figure 3: Routing Protocols

Further, depending upon network structure, three categories of routing protocols are: Flat, Hierarchical and Location Based Routing. Each node in flat routing plays the equal role and they all collaborate with each other to perform the sensing operation. Because of large number of nodes, it is not feasible to assign a global identifier. This led to data centric routing where sink node sends queries to certain regions and waits for data from the sensors located in the selected regions. On the other hand, in hierarchical routing protocols, higher energy nodes act as cluster head and used to process and send the information while nodes with lower energy perform the sensing operation . Constructing clusters and assigning special task to cluster heads can greatly contribute to

Journal of Technology Management for Growing Economies, Volume 6, Number 1, April 2015
Simulating and Analysing the Impact 
Soni, A.

Randhawa, R. overall system scalability, network lifetime and energy efficiency. In Location based routing sensor nodes are addressed by means of their location. Distance between neighbouring nodes can be estimated on the basis of incoming signal strength and their relative coordinates can be obtained by exchanging such information between neighbours. Fig. 4 shows examples of routing protocols of three types.

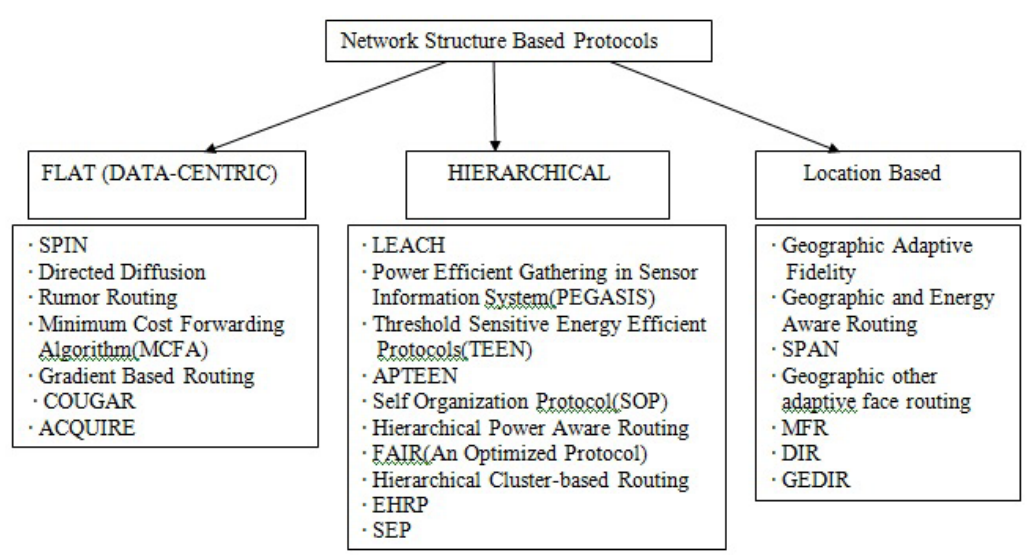

Figure 4: Network Structure Based Routing Protocols

Out of these protocols, we choose 4 hierarchical routing protocols LEACH, SEP, EHRP and FAIR for simulation purposes. All these protocols are based on cluster formation but still having some characteristics which makes them different from each other. LEACH [5] uses random formation of cluster head, so that energy load can be distributed evenly among the sensor nodes. It presumes homogeneous nature of nodes in terms of energy i.e. all the nodes are having same initial energy level. FAIR [6] on the other hand conclude that, if fairness is not taken into consideration among nodes in selection of cluster head than it is possible than some nodes dies before others because of energy drainage. SEP [7] opposing LEACH, explains that it is not always true that all the nodes have same initial energy i.e. nodes can be heterogeneous in terms of energy and therefore cluster head must be elected according to the energy level left in the node. EHRP [8] is also capable of handling heterogeneous energy levels of nodes by formulating a new cluster head selection algorithm in first phase and formation of spanning tree over chosen cluster heads in second phase.

\section{QUANTITATIVE PARAMETERS}

Different parameters of WSNs which are quantitative in nature and can be calculated with the help of proper simulations are : 
Network Lifetime: Network Lifetime is the time interval in which network remains functional starting from deployment. However function of a network depends upon the application for which it has been deployed. In some applications, network is considered failure when the very first node in network dies while in some other applications network remains functional even after some percentage nodes of network dies.

Network Latency: Time taken by a source node to transmit the data to the destination.

Energy Dissipation per Round: It is the amount of energy spent for routing message in one round.

First Dead Node Count: Time when first node in the network dies. In some applications this time is crucial because some applications stops its working even if the single node in the network fails.

Packets to Base Station: Total Number of packets sent to the Base Station successfully.

\section{SIMULATION-SETUP}

Extensive simulations have been done to find the effect of these routing protocols on different parameters using MATLAB. Different modules have been setup for this purpose as shown in table 1:

Table 1: Simulation-Parameters

\begin{tabular}{|c|c|}
\hline \multicolumn{2}{|c|}{ Sensor Deployment Module: } \\
\hline No. of Nodes & 100 \\
\hline Area & $100 * 100 \mathrm{~m}$ \\
\hline Positioning of Nodes & Random \\
\hline Sink Node Position & Centre \\
\hline \multicolumn{2}{|c|}{ Energy Initialization Module: } \\
\hline Initial Energy in Node(Eo) & 0.5 Joules \\
\hline Energy Required for Transmission(ETS) & $10 * 10^{-11}$ Joules \\
\hline Energy Required for Amplification (EMP) & $0.0013 * 10^{-11}$ Joules \\
\hline Data Aggregation Energy (EDA) & $5 * 0.000000001$ Joules \\
\hline \multicolumn{2}{|c|}{ Clustering Module: } \\
\hline Optimal Number of Constructed Clusters (Kopt) & $\begin{array}{l}\text { sqrt(NumberOfNodes / }(2 * \\
\text { pi }) *(2 / 0.765)\end{array}$ \\
\hline $\begin{array}{l}\text { Optimal Election Probability of a Node to } \\
\text { Become Cluster Head(Ep) }\end{array}$ & Kopt / NumberOfNodes \\
\hline Maximum Number of Rounds (RoundMax) & 4000 \\
\hline
\end{tabular}

Journal of Technology Management for Growing Economies, Volume 6, Number 1, April 2015
Simulating and Analysing the Impact 
Soni, A.

Randhawa, R.

\section{RESULTS}
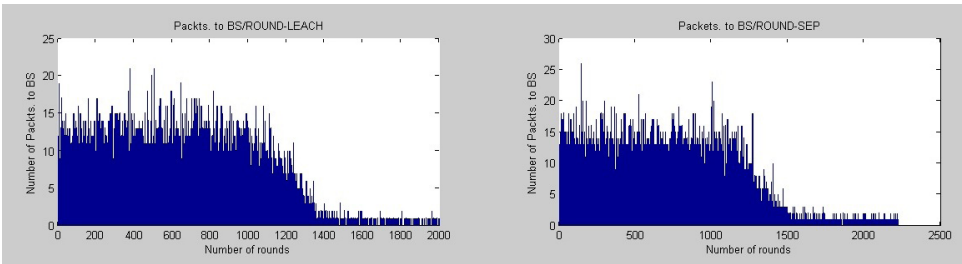

Pockis. to BSROUND-FAIR
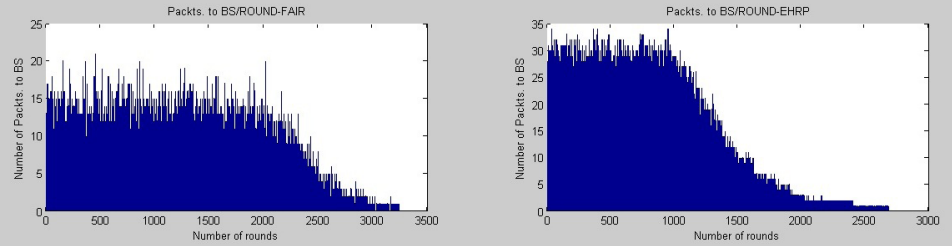

Figure 5: Packets Sent to Base Station

Packets Sent to Base Station: Figure 5 shows that Leach protocols stops sending packets to base station after 2000 rounds and maximum number of packets send in any round are 20. SEP stops it after 2200 rounds and maximum packets sent are 26.In FAIR stops sending packets after 3200 round and maximum packets send are around 20 and in EHRP number of packets sent are approx.32 and it stops sending packets after 2600 rounds.
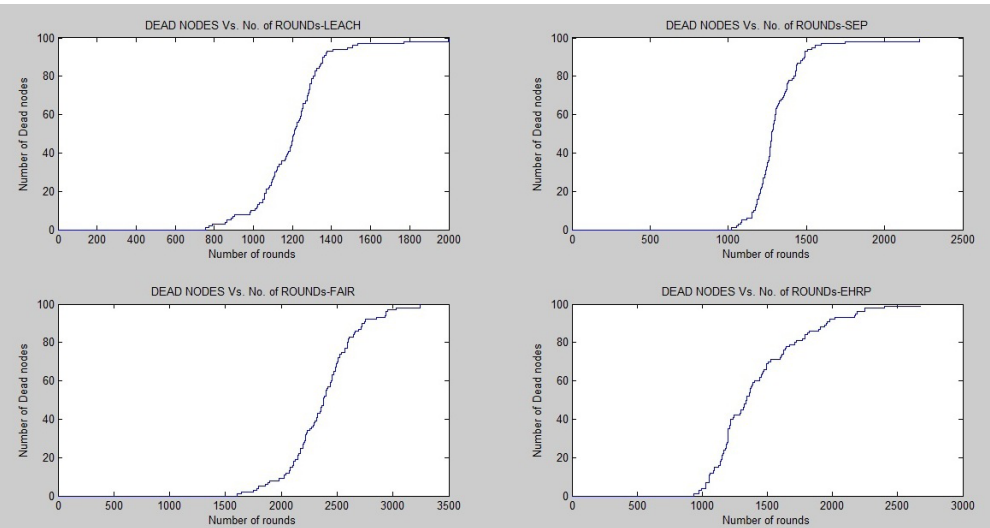

Figure 6: First Dead Node Count

First Dead Node Count: As shown in Figure 6, Nodes in LEACH starts dieing after 800 rounds and all nodes dies in 2000 rounds. In SEP, it starts after 1000 rounds and all nodes dies after 2200 round. In FAIR node, first node dies after 1600 rounds and after 3200 rounds network fails. In EHRP, this count starts after 900 rounds and all nodes dies in 2700 rounds.

Journal of Technology Management for Growing Economies, Volume 6, Number 1, April 2015 

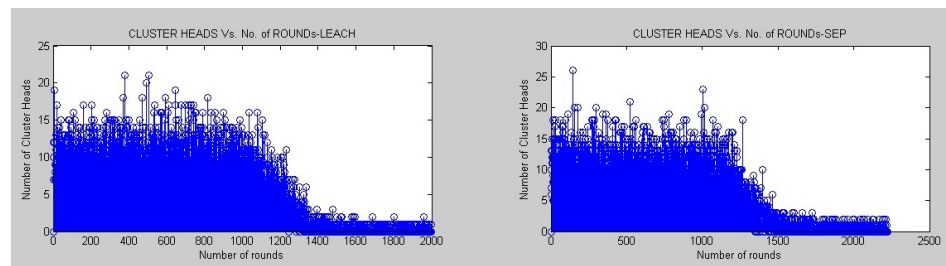

Simulating and

Analysing the

Impact
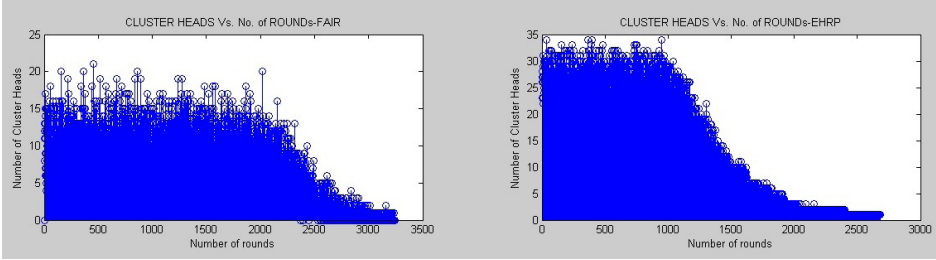

Figure 7: Cluster Head Formed

Cluster Head Formed: As shown in Figure 7, Maximum number of cluster formed in LEACH protocol are 21 and cluster formation continues upto 2000 rounds. In SEP, this number is 27 and cluster formation continues upto 2300 rounds. In FAIR the maximum cluster formed are 20 but cluster formation continues upto more than 3000 rounds. In EHRP maximum cluster formed are more than 30 and this process of cluster formation continues upto 2600 rounds.
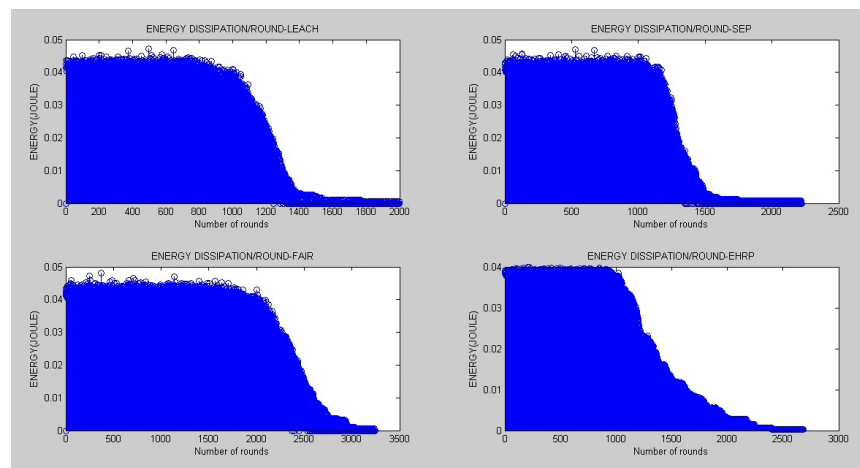

Figure 8: Energy Dissipation

Energy Dissipation(Network Lifetime): As shown in Figure 8, network lifetime of all these protocols increases in LEACH,SEP,EHRP,FAIR sequence means LEACH has the minimum network lifetime while FAIR has the maximum.

Figure 9 shows the comparative analysis for network lifetime and time when first node die. 
Soni, A.

Randhawa, R.

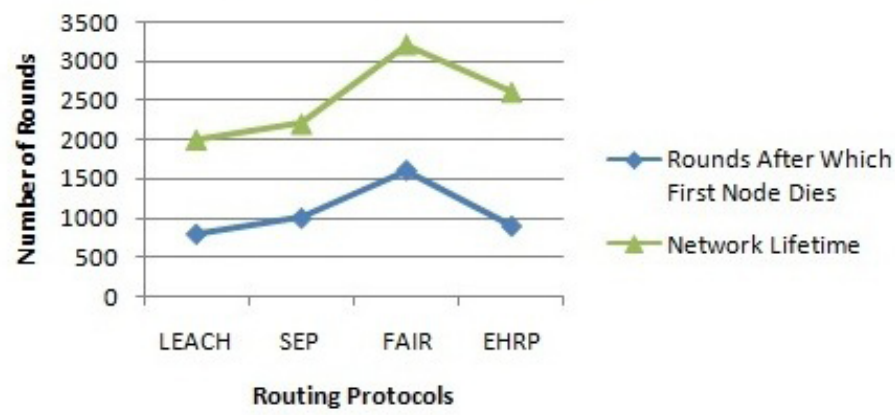

Figure 9: Comparative Analysis of Network Lifetime

Table 2: Comparison of Protocols

\begin{tabular}{|l|l|c|l|l|}
\hline $\begin{array}{l}\text { Routing } \\
\text { Protocol }\end{array}$ & $\begin{array}{l}\text { Cluster } \\
\text { Formation } \\
\text { in Rounds }\end{array}$ & $\begin{array}{l}\text { Heterogeneity } \\
\text { Consideration }\end{array}$ & $\begin{array}{l}\text { Packet } \\
\text { Transmission } \\
\text { to Base Station }\end{array}$ & $\begin{array}{l}\text { Network } \\
\text { Lifetime }\end{array}$ \\
\hline LEACH & Poor & N & Average & Least \\
\hline SEP & Good & Y & Good & Average \\
\hline EHRP & Best & Y & Best & Good \\
\hline FAIR & Good & Y & Good & Best \\
\hline
\end{tabular}

\section{CONCLUSION}

In present work, Four WSN protocols, namely LEACH,SEP,FAIR and EHRP were simulated by using MATLAB. Several tests were carried out using different network parameters of WSNs and finally the effect of these protocols was found on some important parameters. The target was to find out that in any particular application, which routing protocol should be used. So, we conclude that if application depends upon the failure of very first node in the network, than FAIR is suitable because first node dies after a long time as compared to others. Under all the similar circumstances, total network lifetime of FAIR will be greater than others.

\section{REFERENCES}

I.F. Akyildiz et al. (2002) “Wireless Sensor Networks: A survey”, Computer Networks, Vol. 38, No. 4, pp. 393-422.

Raghunathan V., Schurghers C., Park S. and Srivastava M. (2002) "Energy-aware Wireless Microsensor Networks", IEEE Signal Processing Magazine, pp. 40-50. http://dx.doi. org/10.1109/79.985679.

Jamal N. Al-Karaki, Ahmed E. Kamal (2004) “Routing Techniques in Wireless Sensor Networks: A Survey", IEEE Wireless Communications.

Shio Kumar Singh, M P Singh, D K Singh (2010) "Routing Protocols in Wireless Sensor Networks - A Survey”, International Journal of Computer Science \& Engineering Survey

Journal of Technology Management for Growing Economies, Volume 6, Number 1, April 2015 
(IJCSES) Vol.1, No.2.

W. Heinzelman, A. Chandrakasan, H. Balakrishnan (2000) "Energy-efficient Routing Protocols for Wireless Microsensor Networks" in 33rd Hawaii International Conference of System Sciences (HICSS), Maui, HI. http://dx.doi.org/10.1109/HICSS.2000.926982.

Amir Hossein, Mohammad Hossein, Hadi Sadoghi, Abbas Ali Rezaee (2009) "A Fair Routing Protocol Using Generic Utility Based approach in Wireless Sensor Networks", In International Conference on ultra modern Telecommunications \& Workshop. http:// dx.doi.org/10.1109/ICUMT.2009.5345348.

Georgios Smaragdakis, Ibrahim Matta, Azer Bestavros (2004) "Sep: A Stable Election Protocol for Clustered Heterogeneous Wireless Sensor Networks", in Second International Workshop on Sensor and Actor Network Protocols and Applications.

Amir Mollanejad, Leili Mohammad Khanli, Mohammad Zeynali,Hadi Bahrbegi (2010) "EHRP: Novel Energy-Aware Hierarchical Routing Protocol in Wireless Sensor Network" in IEEE Ultra Modern Telecommunications and Control Systems and Workshops (ICUMT), 18-20. http://dx.doi.org/10.1109/ICUMT.2010.5676492.
Simulating and Analysing the Impact 